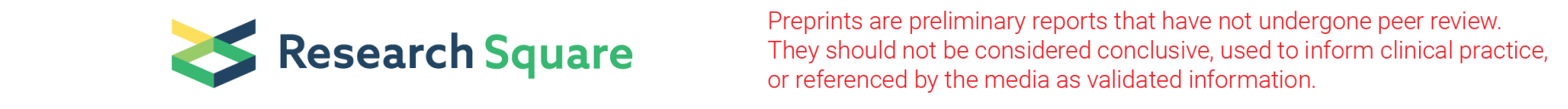

\title{
Predictors of Prognosis of Surgically Resected Brain Metastasis from Lung Cancer with a Focus on Brain Recurrence
}

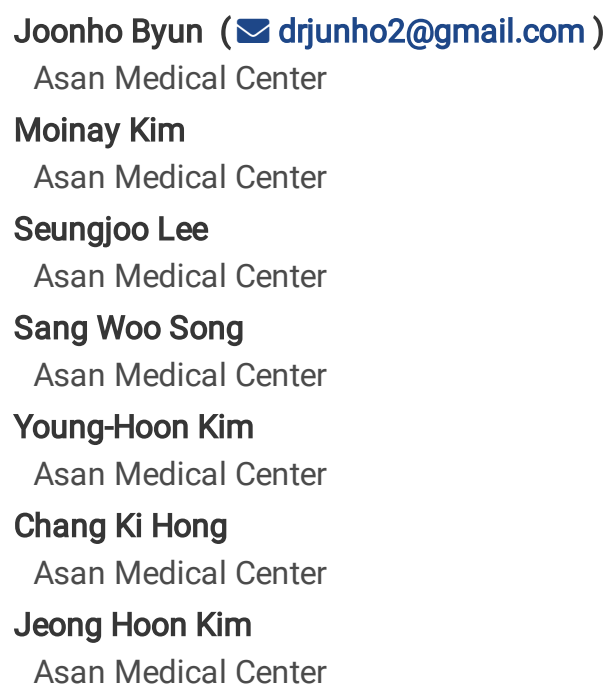

\section{Research Article}

Keywords: Lung cancer, brain metastasis, surgery, recurrence, survival

Posted Date: September 16th, 2021

DOI: https://doi.org/10.21203/rs.3.rs-853199/v1

License: (c) (i) This work is licensed under a Creative Commons Attribution 4.0 International License. Read Full License 


\section{Abstract}

Purpose: There are many considerations to decide surgical treatment in lung cancer-brain metastasis. Herein, we analyzed survival and predictors of brain recurrence after surgery for lung cancer-brain metastasis.

Methods: A total of 224 metastatic brain tumors from patients with lung cancer were analyzed.

Results: There were 138 (61.6\%) male and 86 (38.4\%) female patients; 197 patients with non-small cell lung cancer (NSCLC) and 27 with small cell lung cancer (SCLC). The rate of postoperative complications was $4.9 \%$. The 1-year and 2-year overall survival (OS) was $57 \%$ and $43 \%$, respectively. The 6-month and 1-year progression-free survival (PFS) of local recurrence was $42 \%$ and $20 \%$, respectively. In NSCLC and SCLC groups, the difference of PFS of local recurrence according to adjuvant irradiation was not significant (1-year: $20 \%$ vs. $22 \%, p=0.8$ ) and (1-year: $14 \%$ vs. $25 \%, p=0.16$ ), respectively. Whole-brain irradiation did not show a significant difference of distant recurrence (6-month: $54 \%$ vs. $60 \%, p=0.13$ ). In predictor analysis, female sex was a favorable prognostic factor for OS, while SCLC histology and postoperative neurological deficits were poor prognostic factors in univariate analysis.

Conclusions: SCLC histology and postoperative complications are negative predictors for OS. Adjuvant irradiation did not show effectiveness to reduce local and distant recurrence.

\section{Introduction}

Lung cancer is the most common cause of cancer-related death in South Korea ${ }^{1}$. Brain metastasis is a major concern in the treatment of lung cancer. Sometimes, lung cancer is retrospectively diagnosed from brain metastatic mass. From the previous report, the incidence of brain metastasis in patients with primary lung cancer was $20 \%{ }^{2}$. Additionally, it has been reported that the incidence of lung cancer brain metastasis has been increasing in recent years, which places a great burden on public health services ${ }^{3}$. The treatment modalities of brain metastasis are surgery, conventional radiation therapy, systemic chemotherapy, and stereotactic radiosurgery (SRS). Recently, SRS including gamma-knife and cyber-knife radiosurgery has shown good results for local control for single and multiple brain metastasis; however, surgical resection still plays an important role. Surgery provides immediate relief of mass effect, obtaining tissue for histopathologic examination. In particular, surgical resection is the essential treatment in large metastasis. There are many considerations to decide surgical treatment in patients with lung cancer-brain metastasis, such as current disease state of lung cancer, performance status, tolerability for general anesthesia and craniotomy, expected survival after surgery, and postoperative neurological deficits. According to previously published studies, the median survival of lung cancer brain metastasis was reported at 3-12 months ${ }^{4-}$

7. Considering short survival and quality of life of patients with cancer, prediction of recurrence and survival is important during surgical decision-making. From a neurosurgeon's perspective, minimizing local and distant brain recurrence after surgery of metastatic tumor is the critical consideration and always have a question for adjuvant treatment after total resection of metastatic tumor. Herein, we investigated predictors of recurrence and survival of surgically treated brain metastasis from lung cancer.

\section{Methods}

\subsection{Patient selection}

This study was approved by the institutional review board of Asan Medical Center. Our institutional database was searched for metastatic brain tumors from lung cancer diagnoses between January 2010 and December 2020. Only newly diagnosed cases and patients who underwent microsurgery for lung cancer brain metastasis at our institute were enrolled in the current study; recurrent tumors with no available information, brain metastasis from other primary tumors were excluded. A total of 224 patients with metastatic brain tumors from lung cancer treated by surgical resection and confirmed on histopathological examination were initially included in the study population. The tumor size was defined by its maximal diameter in two dimensions.

\subsection{Treatment and follow-up}

Treatment decision of lung cancer brain metastasis has been established in "Integrated lung cancer treatment team," comprising oncologists, radiation-oncologists, and neurosurgeons. The extent of resection in our study was re-defined as follows: gross total resection, complete removal of the tumor as indicated on postoperative magnetic resonance imaging (MRI); subtotal resection, any incomplete resection of the tumor with less than $10 \%$ of the tumor visible as a remnant on postoperative MRI. SRS including gammaknife (GK) radiosurgery (RS) and cyber-knife (CK) RS were additionally performed for residual tumors. GKRS was performed using a 
Leksell GK perfexion (December 2011 until the present). Dose planning for the GKRS treatments used MRI analysis in the Leksell gamma plan. The Elekta and marginal prescription doses were from 20 to $24 \mathrm{~Gy}$. The Robotic Radiosurgery System Version 9.0 was used for CK treatments. For CK planning, computed tomography images of a very thin slice ( $0.625 \mathrm{~mm}$ thickness with no slice gap) were obtained and fused with gadolinium-enhanced axial and coronal three-dimensional T1-magnetization-prepared rapid acquisition gradient-echo MR images (1.0 mm slice) in the Accuray MultiPlan system (version 4.5). The median prescription dose was $35 \mathrm{~Gy}$ (range: 25-41 Gy), the dose was administered in three or five daily fractions. Conventional radiation therapy was administered for the surgical cavity or whole brain, the prescription dose was 30 Gy and was fractionated by 2 or 3 Gy.

Initial follow-ups involved clinical evaluations and an MRI in the immediate postoperative period (within $48 \mathrm{~h}$ ), and at 1 and 3 months, and then checked every 3 months.

\subsection{Statistical analysis}

Subgroup comparisons were performed using a Student's $t$-test, Chi-square test, or Fisher's exact tests. Overall survival (OS) and progression-free survival (PFS) were evaluated in addition to prognostic factors. The OS and PFS outcomes were analyzed using Kaplan-Meier survival analysis, and subgroup comparisons were performed using log-rank tests. Potential prognostic factors for PFS, including age, sex, multiple metastases, adjuvant resection bed irradiation, adjuvant whole-brain radiation therapy (WBRT), the period from lung cancer diagnosis to brain metastasis, postoperative neurological sequelae, immunohistochemical findings in NSCLC were analyzed using a Cox-proportional hazards model. The proportional hazards assumption was confirmed by the testing of Schoenfeld residuals, and no relevant violations were found. All statistical analyses were conducted using SPSS ver. 21.0 (IBM Corp., Armonk, NY). A $p$-value $<0.05$ was considered statistically significant.

\section{Results}

\subsection{Study patient characteristics and treatment outcomes}

There were 138 (61.6\%) male and 86 (38.4\%) female patients with a median age of 60 years. There were 197 patients with non-small cell lung cancer (NSCLC) and 27 with small cell lung cancer (SCLC). Among patients with NSCLC, 52.3\% patients were epidermal-growth factor receptor (EGFR)-mutant, and $47.7 \%$ were EGFR non-mutant. Among patients with NSCLC, the initial stage was stage 4 in $54.6 \%$ and extended disease among $88.8 \%$ of SCLC patients. The most common location was the frontal lobe (32.6\%) following the cerebellum $(17.9 \%)$ and temporal lobe (14.3\%). The representative image findings were presented in Figure 1. One hundred five (73.7\%) patients had single brain metastasis and $59(26.3 \%)$ had multiple brain metastasis. The maximal diameter was $42 \mathrm{~mm}$. The median period from lung cancer diagnosis to brain metastasis was 8 months; however, one patient showed 226 months recurrence in the brain from complete remission of lung cancer. The rate of postoperative complications was $4.9 \%$. Twenty-eight patients (12.5\%) received conventional radiation therapy and $49(21.8 \%)$ patients received SRS. The median follow-up period was 12 months. The detailed characteristics were presented in Table 1.

\subsection{Progression-free and overall survival between groups}

The 1-year and 2-year OS rates of patients with lung cancer brain metastasis were $57 \%$ and $43 \%$, respectively. The 6 -month and 1-year PFS of local recurrence was $42 \%$ and $20 \%$, respectively and distant recurrence was $54 \%$ and $24 \%$, respectively. (Figure 2 ). In terms of Recursive Partitioning Analysis (RPA) classification, RPA class 1 group showed longer survival compared to class 2 and 3 groups $(p=0.04)$.

NSCLC vs. SCLC: The 1-year and 2-year OS of NSCLC was 58\% and 42\%, respectively, and the 1 -year and 2-year OS of SCLC was $44 \%$ and $10 \%$, respectively. The difference of OS between NSCLC and SCLC was statistically significant $(p=0.01)$. The 6-month and 1-year PFS of local recurrence of NSCLC was $51 \%$ and $20 \%$, respectively and SCLC was $36 \%$ and $18 \%$ months, respectively $(p=0.35)$. The 6month and 1-year PFS of distant recurrence of NSCLC were $56 \%$ and $19 \%$, and SCLC was $56 \%$ and $18 \%$, respectively. (Figure 2)

Adjuvant resection bed irradiation and WBRT: In the NSCLC group, the difference of PFS of local recurrence according to adjuvant resection bed irradiation was not significant (6-month: $38 \%$ vs. $66 \%, 1$-year: $20 \%$ vs. $22 \%, p=0.8$ ). In the SCLC group, the difference of PFS of local recurrence according to adjuvant resection bed irradiation was also not significant (6-month: $14 \%$ vs. $75 \%, 1$-year: $14 \%$ vs. $25 \%, p=0.16)$. Whole-brain irradiation did not show a significant difference in PFS of distant recurrence (6-month: $54 \%$ vs. $60 \%, p=0.13)$. Leptomeningeal seeding occurred in $38(17 \%)$ patients during treatment, WBRT group did not show superiority for leptomeningeal seeding (l-year PFS rate: non-WBRT group= $85.9 \%$, WBRT group $=81.8 \%, p=0.87$ ). (Figure 3 ) 
Postoperative complications: The postoperative complications occurred in 11 patients. Patients who experienced postoperative complications showed statistically significant shorter OS than those who did not experience postoperative complications ( 1 year: $59 \%$ vs.27\%, $p=0.03$ ). (Figure 3-[D])

Immunohistochemical findings: EGFR, anaplastic lymphoma kinase-1 (ALK-1), programmed death ligand-1 (PD-L1) are known as the therapeutic target of NSCLC. In terms of EGFR, EGFR mutation did not show significant difference of PFS for local recurrence (6-month: $29 \%$ vs. $54 \%$, 1 -year: $11 \%$ vs. $25 \%, p=0.16$ ) and distant recurrence (6-month: $56 \%$ vs. $56 \%, 1$-year: $25 \%$ vs. $18 \%, p=0.22$ ). However, the EGFR mutant group showed statistically significant longer OS than EGFR non-mutant group (2-year: 38\% vs.53\%, p=0.04) (Figure 3). ALK mutation and PD L1 mutation did not show significant differences in PFS (local recurrence and distant recurrence) and OS. (Figure 4)

\subsection{Prognostic indicators of progression-free survival and overall survival}

We analyzed predictors of local, distant recurrence and OS including age, sex, multiple metastases, SCLC histology, resection bed irradiation, WBRT, the period from lung cancer diagnosis to brain metastasis shorter than 12 months, postoperative neurological deficits, and immunohistochemical findings (EGFR mutation, ALK-1 mutation, and PD-L1 mutation). In univariate analysis, female sex was a favorable prognostic factor for PFS for distant recurrence, as well as for OS; however, SCLC histology and postoperative neurological deficits were negative predictors for OS. In multivariate analysis, ALK-1 mutation showed intermediate significance for local recurrence. There was no significant predictor for distant recurrence. Female patient and postoperative neurological deficits were statistically significant predictors for OS ( $\mathrm{HR}=0.5$ [95\% Cl: $0.3-0.7, \mathrm{p}=0.01]$ and $\mathrm{HR}=2.46[95 \% \mathrm{Cl}=1.28-7.72, \mathrm{p}=0.01])$. Detailed analysis was presented in Table 2.

\section{Discussion}

Lung cancer is the most frequent source of metastatic brain tumors. It occurs in $17-65 \%$ of patients with primary lung cancer ${ }^{3,8}$. The rate of lung cancer brain metastasis has been increasing in recent years ${ }^{3}$. According to previously published studies, the prognosis for patients with lung cancer brain metastasis is poor, median OS of lung cancer brain metastasis was reported from 1 to 12 months despite treatment ${ }^{4-6}$. However, there are significant advances in the management of lung cancer, to our study, the median OS was over 12 months.

To prolong the survival and improve the quality of life of lung cancer brain metastasis, neurological symptom and relief intracranial hypertension, the treatment of brain metastasis is critical in patients with lung cancer. Thus, the role of a neurosurgeon is considerably important in the treatment of lung cancer brain metastasis. From the perspective of a neurosurgeon in brain metastasis treatment, decreasing local and distant recurrence with maintaining or improving the life of quality are the most important considerations. Surgery, stereotactic radiosurgery, conventional radiation therapy, and systemic chemotherapy are the possible treatment strategies in brain metastasis. The efficacy of radiosurgery for small-sized oligometastasis has been proved ${ }^{7}$. Moreover, Chon et al. reported the feasibility of fractionated radiosurgery for medium-sized brain metastasis ${ }^{9}$. However, for medium to large tumors, surgical resection is still an essential tool. Moreover, surgery is the only option for large tumors with mass effect, establishing pathological confirmation, progression after radiosurgery, or radiotherapy. Recently, we have performed surgical resection at our institute of not only single metastasis or oligo-metastasis but also multiple metastases. Surgery with adjunctive radiosurgery or radiotherapy were performed to prolong survival or relieving symptoms related to brain metastasis.

In this study, we focused on analyzing predictors for local and distant recurrence of the brain from a neurosurgeon's perspective. Total resection of metastatic brain tumor was performed for $97 \%$ of patients. To reduce local recurrence, "en-bloc"-resection showed a better outcome than "piecemeal resection" ${ }^{10}$. Furthermore, Yoo et al. reported plus $5 \mathrm{~mm}$ adjacent white matter resection reduced local recurrence ${ }^{11}$. In our series, we did gross total resection without plus normal tissue resection. Although, all tumors could not be feasible, to reduce local recurrence rate, "en-bloc"-fashion resection plus white matter is pursued for non-eloquent area tumors.

Postoperative radiotherapy and SRS for reducing local recurrence could be applied in the resection cavity. SRS and WBRT showed comparable results for local recurrence in brain metastasis ${ }^{6,12}$ In the previous report, the efficacy of SRS and WBRT are comparable $6,12,13$. WBRT has been needed for disseminated metastasis and it harbored a unique role, however, considering the neuro-cognitive complications including learning and memory dysfunction, SRS may be a more suitable tool for resection bed irradiation ${ }^{14}$. 
Theoretically, resection bed irradiation may provide good local control, but poor distant recurrence control. However, in our series, resection bed radiotherapy and radiosurgery did not affect the local recurrence of both NSCLC and SCLC brain metastasis. Additionally, postoperative WBRT failed to affect the distant recurrence. It implicated adjuvant radiotherapy and radiosurgery may not be mandatory after surgical resection for lung cancer brain metastasis. In the previous studies, only WBRT for brain metastasis impact survival from 1 to 3 months ${ }^{15,16}$. Applying WBRT for reducing distant recurrence in lung cancer brain metastasis considering its survival benefit and neurocognitive impairment is still debatable. According to our result, WBRT did not improve the PFS for distant recurrence.

At our institute, there was no consensus for applying adjuvant radiotherapy or SRS, it depends on the opinion of individual oncologists. Standard cytotoxic chemotherapy used in lung cancer, mainly platinum agents is not effective for lung cancer brain metastasis owing to poor penetration of the blood-brain barrier ${ }^{4}$. Molecular targeted therapy showed the promising effect of lung cancer-NSCLC brain metastasis ${ }^{4,17}$. EGFR mutation, ALK rearrangement, and PD-L1 are targetable mutations. They have shown effectiveness in intracranial disease control; moreover, a recent clinical trial reported the promising result of EGFR-targeted therapy for the leptomeningeal disease ${ }^{18}$. However, still, they are not suitable for symptomatic and immediate life-threatening brain metastasis, it could be applied for small metastasis and disseminated disease. At our institute, we have used a molecular targeted agent after surgical resection of metastatic brain tumor according to the status of targetable gene mutation. We evaluated the difference of local recurrence and distant recurrence concerning EGFR, ALK-1, and PD-L1 in the NSCLC group. There was no statistically significant difference in local and distant recurrence. EGFR mutant NSCLC brain metastasis group showed superior OS rate, which may be attributed to extracranial disease control by EGFRtarget agent. The efficacy of molecular target agent after brain metastatic tumor resection should be evaluated at the future study.

The maintenance of the quality of life of patients with cancer is an important consideration. Considering the lower chance of cure of disease in patients with lung cancer brain metastasis, the postoperative complication significantly impacting the performance status of patients should be minimized. In recent studies, the eloquent location of metastatic brain tumors could be managed using radiosurgery

7,9 . Declining of performance status due to postoperative neurological deficits not only restrict further chemotherapy or radiation therapy but also leads to other medical complications, which jeopardize the survival of patients with lung cancer. In our study, the postoperative complication group showed a significantly lower OS rate. We should minimize the postoperative complication in lung cancer brain metastasis surgery, and consider that the complication critically influences survival.

\section{Study limitations and strengths}

Our current study had some limitations. In the first instance, it was a retrospective investigation that included 224 patients with lung cancer brain metastasis. Along with the limitations inherent to any retrospective design, this precluded less meaningful multivariate analysis of survival outcomes or predictors of local and distant recurrence. This study series spanned around 10 years; therefore, the treatment methods varied. Additionally, the management strategy of lung cancer brain metastasis was varied according to individual neurosurgeons, which made it challenging to conclude on the optimal intervention strategies and outcomes in these cases.

Our study also had some notable strengths. However, including our finding that the SCLC and postoperative complication groups have a significantly shorter OS than the adjuvant resection bed RT and SRS, usage of targeted therapy after resection may not be a predictive factor for local and distant recurrence in lung cancer brain metastasis. Although we could not draw any meaningful conclusions to assist with future treatment guidelines, our analysis indicates that female sex, SCLC histology, and postoperative complications are prognostic indicators in terms of survival, and require careful consideration to improve survival and maintain the quality of life in metastasis. Therefore, we believe that the data from our current single-center series make a valuable contribution to the available literature on these extremely rare tumors and to a future meta-analysis of this disease.

\section{Conclusion}

Surgery of lung cancer brain metastasis is an essential treatment tool to improve survival and defer life-threatening events during lung cancer treatment. SCLC histology and postoperative complication are negative predictors for OS. Adjuvant SRS, RT, and targeted chemotherapy do not show effectiveness to reduce local and distant recurrence. However, a future large-sized study should be conducted.

\section{Declarations}

\section{Acknowledgement}


The authors thank the oncologists and radiation-oncologists our institute. We also thank Prof. Min Jae Yoon, a Neuro-radiologists of CHA medical center in Gumi City, who gave us valuable advice and help.

\section{Authors' contributions}

Author contributions JB and YHK conceived and designed the study. MK and SL conducted the literature search. JB, MK, YHK, SWS and $\mathrm{CKH}$ were involved in the analysis and interpretation of data. JB drafted the manuscript. The study was supervised by CKH and JHK. All authors read and approved the final manuscript.

The authors declare no competing interests regarding this study.

\section{Funding}

This research did not receive any specific grant from funding agencies in the public, commercial, or not-for-profit sectors.

\section{Ethics approval}

This retrospective study has been approved by the appropriate ethics committee and was performed in accordance with the ethical standards laid down in the 1964 Declaration of Helsinki and its later amendments. (Approval by IRB of Asan Medical Center, No. 20211137). For this type of study, formal patient consent is not required. The IRB of Asan Medical Center gave the right of exemption of 'informed consent' for this study. The reason described below.

The risk expected from this 'retrospective observational study' is not greater than the Level I risk. In addition, all of included patients' dataset were anonymized. There is no reason to presume the refusal of the research subject's consent, and the risk to the enrolled patients is extremely low even if consent is waived. Consent exemption does not infringe on the rights or welfare of the subject. It is not the research for drug/medical device approval, and it is not a research regulated by a foreign regulatory agency.

\section{References}

1. Jung, K.-W., Won, Y.-J., Kong, H.-J. \& Lee, E. S. Cancer Statistics in Korea: Incidence, Mortality, Survival, and Prevalence in 2016. Cancer Reserach and Treatment 51, 417-430 (2019).

2. Barnholtz-Sloan, J. S. et al. Incidence Proportions of Brain Metastases in Patients Diagnosed (1973 to 2001) in the Metropolitan Detroit Cancer Surveillance System. Journal of Clinical Oncology 22, 2865-2872 (2004).

3. Fox, B. D., Vincent J. Cheung, Patel, A. J., Suki, D. \& Rao, G. Epidemiology of Metastatic Brain Tumors. Neurosurg Clin N Am 22, 1-6 (2011).

4. Abdallah, S. M. B. \& Wong, A. Brain metastases in non-small-cell lung cancer: are tyrosine kinase inhibitors and checkpoint inhibitors now viable options? Current Oncology 25, S103-S114 (2018).

5. Mulvenna, P. et al. Dexamethasone and supportive care with or without whole brain radiotherapy in treating patients with non-small cell lung cancer with brain metastases unsuitable for resection or stereotactic radiotherapy (QUARTZ): results from a phase 3, noninferiority, randomised trial. Lancet $\mathbf{3 8 8}$ (2016).

6. Lamba, N. et al. Stereotactic radiosurgery versus wholebrain radiotherapy after intracranial metastasis resection: a systematic review and meta-analysis. Radiation Oncology 12, 106 (2017).

7. Fuentes, R., Cosp, X. B. \& Hernandez, J. E. Surgery versus radiosurgery for patients with a solitary brain metastasis from non-small cell lung cancer (Review). Cochrane Library (2006).

8. Gavrilovic, I. T. \& Posner, J. B. Brain metastases: epidemiology and pathophysiology. Journal of Neuro-Oncology 75, 5-14 (2005).

9. Chon, H., Yoon, K., Lee, D., Kwon, D. H. \& Cho, Y. H. Single-fraction versus hypofractionated stereotactic radiosurgery for medium-sized brain metastases of 2.5 to $3 \mathrm{~cm}$. Journal of Neuro-Oncology 145, 49-56 (2019).

10. Patel, T. R., Knisely, J. P. S. \& Chiang, V. L. S. Management of Brain Metastases Surgery, Radiation, or Both? Hematol Oncol Clin N Am 26, 933-947 (2012).

11. Yoo, H. et al. Reduced local recurrence of a single brain metastasis through microscopic total resection. Journal of Neurosurgery 110, 730-736 (2009).

12. Putz, F. et al. FSRT vs. SRS in Brain Metastases-Differences in Local Control and Radiation Necrosis-A Volumetric Study. Radiation Oncology 10, 559193 (2020). 
13. Ahmed, Z. et al. Postoperative stereotactic radiosurgery for resected brain metastasis. CNS oncology 3, 199-207 (2014).

14. Chang, E. L. et al. Neurocognition in patients with brain metastases treated with radiosurgery or radiosurgery plus whole-brain irradiation: a randomised controlled trial. Lancet Oncology 10, 1037-1044 (2009).

15. Khuntia, D., Brown, P., Li, J. \& Mehta, M. P. Whole-Brain Radiotherapy in the Management of Brain Metastasis. Journal of Clinical Oncology 24, 1295-1304 (2006).

16. Lu-Emerson, C. \& Eichler, A. F. Brain Metastases. Continuum Lifelong Learning Neurol 18, 295-311 (2012).

17. Zer, A. \& Leighl, N. Promising targets and current clinical trials in metastatic non-squamous NSCLC. Frontier in Oncology 4 (2014).

18. Ahn, M.-J. et al. Activity and safety of AZD3759 in EGFR-mutant non-small-cell lung cancer with CNS metastases (BLOOM): a phase 1, open-label, dose-escalation and dose-expansion study. Lancet 5, 891-902 (2017).

\section{Tables}

Table 1. Basal characteristics of enrolled patients 


\begin{tabular}{|c|c|}
\hline & Total N=224 \\
\hline Age & Median 60 (27-79) \\
\hline \multicolumn{2}{|l|}{ Sex } \\
\hline Male & $138(61.6 \%)$ \\
\hline Female & $86(38.4 \%)$ \\
\hline \multicolumn{2}{|l|}{ Histology } \\
\hline NSCLC & $197(87.9 \%)$ \\
\hline Squamous & 37 \\
\hline Adenocarcinoma & 145 \\
\hline Others & 15 \\
\hline NSCLC-EGFR mutant & $52.3 \%$ \\
\hline NSCLC-EGFR non mutant & $47.7 \%$ \\
\hline Small cell carcinoma & $27(12.1 \%)$ \\
\hline \multicolumn{2}{|l|}{ Initial lung cancer stage } \\
\hline \multicolumn{2}{|l|}{ NSCLC } \\
\hline I & 33 \\
\hline$\|$ & 15 \\
\hline III & 41 \\
\hline IV & 107 \\
\hline SCLC & Total $=27$ \\
\hline Limited & 3 \\
\hline Extended & 24 \\
\hline \multicolumn{2}{|l|}{ Location of tumor } \\
\hline Frontal & $73(32.6 \%)$ \\
\hline Parietal & $56(25 \%)$ \\
\hline Temporal & $32(14.3 \%)$ \\
\hline Occipital & $22(9.8 \%)$ \\
\hline Cerebellum & $40(17.9 \%)$ \\
\hline Others & $1(0.4 \%)$ \\
\hline Single brain metastasis & $165(73.7 \%)$ \\
\hline Multiple brain metastasis & $59(26.3 \%)$ \\
\hline Period from lung cancer diagnosis to brain metastasis (months, median) & $8(0-226)$ \\
\hline \multicolumn{2}{|l|}{ Extracranial metastasis } \\
\hline Absence & $159(71 \%)$ \\
\hline Presence & $65(29 \%)$ \\
\hline \multicolumn{2}{|l|}{ RPA class } \\
\hline Class 1 & $28(12.5 \%)$ \\
\hline Class 2 & $108(48.2 \%)$ \\
\hline
\end{tabular}




\begin{tabular}{|l|l|}
\hline Class 3 & $88(39.3 \%)$ \\
\hline Maximal diameter of tumor (mm, median) & $42(10-80)$ \\
\hline Total resection & $218(97.3 \%)$ \\
Subtotal resection & $6(2.7 \%)$ \\
\hline Postoperative complication & $11(4.9 \%)$ \\
ICH & 6 \\
EDH & 2 \\
\hline Infarction & 3 \\
\hline Adjuvant radiotherapy & $28(12.5 \%)$ \\
Conventional RT & 11 \\
- Resection bed & 17 \\
- Whole brain & $12(0-119)$ \\
\hline Stereotactic radiosurgery & $101(45.3 \%)$ \\
- Resection bed & $71(31.7 \%)$ \\
- Another metastatic lesion & 30 \\
\hline Adjuvant CTX & \\
\hline Non-target agents & \\
\hline Target agents & \\
\hline
\end{tabular}

NSCLC: non-small cell lung cancer, SCLC: small cell lung cancer, EGFR: epidermal growth factor receptor, EDH: epidural hemorrhage, ICH: intracerebral hemorrhage, RT: radiation therapy, CTX: chemotherapy, KPS: Karnofsky Performance Scale, RPA: recursive partitioning analysis

Table 2. Predictors of prognosis of surgically resected lung cancer brain metastasis (Cox-proportional hazard analysis) 


\begin{tabular}{|c|c|c|c|c|c|c|c|c|c|c|c|c|}
\hline & \multicolumn{6}{|c|}{ Univariate analysis } & \multicolumn{6}{|c|}{ Multivariate analysis } \\
\hline & \multicolumn{2}{|c|}{$\begin{array}{l}\text { PFS (Local } \\
\text { recur) }\end{array}$} & \multicolumn{2}{|c|}{$\begin{array}{l}\text { PFS (Distant } \\
\text { recur) }\end{array}$} & \multicolumn{2}{|c|}{ Overall survival } & \multicolumn{2}{|c|}{$\begin{array}{l}\text { PFS } \\
\text { (local recur) }\end{array}$} & \multicolumn{2}{|c|}{$\begin{array}{l}\text { PFS (distant } \\
\text { recur) }\end{array}$} & \multicolumn{2}{|c|}{ Overall survival } \\
\hline & $\begin{array}{l}\text { HR } \\
(95 \% \\
\text { Cl) }\end{array}$ & $\begin{array}{l}\mathrm{P} \text { - } \\
\text { value }\end{array}$ & $\begin{array}{l}\mathrm{HR} \\
(95 \% \\
\mathrm{Cl})\end{array}$ & $\begin{array}{l}\text { P- } \\
\text { value }\end{array}$ & $\begin{array}{l}\text { HR } \\
\text { (95\% } \\
\text { Cl) }\end{array}$ & $\begin{array}{l}\mathrm{P} \text { - } \\
\text { value }\end{array}$ & $\begin{array}{l}\text { HR } \\
(95 \% \\
\text { Cl) }\end{array}$ & $\begin{array}{l}\mathrm{P}- \\
\text { value }\end{array}$ & $\begin{array}{l}\text { HR } \\
(95 \% \\
\text { Cl) }\end{array}$ & $\begin{array}{l}\mathrm{P} \text { - } \\
\text { value }\end{array}$ & $\mathrm{HR}(95 \% \mathrm{Cl})$ & $\begin{array}{l}\mathrm{P} \text { - } \\
\text { value }\end{array}$ \\
\hline \multirow{2}{*}{$\begin{array}{l}\text { Age } \\
>60 \text { years }\end{array}$} & & & 1.15 & 0.52 & 1.22 & 0.22 & NA & & & & & \\
\hline & $\begin{array}{l}1.13 \\
(0.67- \\
1.89)\end{array}$ & 0.63 & $1.81)$ & & $\begin{array}{l}1.69) \\
1.69)\end{array}$ & & & & & & & \\
\hline \multirow{2}{*}{$\begin{array}{l}\text { Sex } \\
\text { Female }\end{array}$} & & & & & & & 0.66 & 0.13 & 0.69 & 0.15 & $0.50(0.35-$ & 0.01 \\
\hline & $\begin{array}{l}0.68 \\
(0.40- \\
1.16)\end{array}$ & 0.16 & $\begin{array}{l}0.63 \\
(0.40- \\
1.00)\end{array}$ & 0.05 & $\begin{array}{l}0.47 \\
(0.33- \\
0.67)\end{array}$ & 0.01 & $\begin{array}{l}\left(.30^{-}\right. \\
1.12)\end{array}$ & & $1.13)$ & & & \\
\hline $\begin{array}{l}\text { Multiple } \\
\text { metastasis }\end{array}$ & $\begin{array}{l}0.89 \\
(0.51- \\
1.54)\end{array}$ & 0.68 & $\begin{array}{l}0.49 \\
(0.94- \\
2.36)\end{array}$ & 0.08 & $\begin{array}{l}1.31 \\
(0.91- \\
1.87)\end{array}$ & 0.13 & NA & & $\begin{array}{l}1.39 \\
(0.87- \\
2.22)\end{array}$ & 0.16 & $\begin{array}{l}1.31(0.91- \\
1.89)\end{array}$ & 0.13 \\
\hline \multicolumn{3}{|l|}{ NSCLC } & & & & & \multirow{2}{*}{\multicolumn{2}{|c|}{ NA }} & & & & \\
\hline SCLC & $\begin{array}{l}1.27 \\
(0.63- \\
2.53)\end{array}$ & 0.49 & $\begin{array}{l}1.38 \\
(0.79- \\
2.42)\end{array}$ & 0.24 & $\begin{array}{l}1.83 \\
(1.15- \\
2.91)\end{array}$ & 0.01 & & & $\begin{array}{l}1.15 \\
(0.63- \\
2.12)\end{array}$ & 0.63 & $\begin{array}{l}1.51(0.94- \\
2.43)^{-}\end{array}$ & 0.08 \\
\hline $\begin{array}{l}\text { Adjuvant } \\
\text { resection bed } \\
\text { irradiation }\end{array}$ & $\begin{array}{l}0.82 \\
(0.44- \\
1.53)\end{array}$ & 0.54 & NA & & $\begin{array}{l}0.96 \\
(0.64- \\
1.45)\end{array}$ & 0.86 & NA & & NA & & NA & \\
\hline $\begin{array}{l}\text { Adjuvant } \\
\text { WBRT }\end{array}$ & NA & & $\begin{array}{l}0.93 \\
(0.76- \\
4.88)\end{array}$ & 0.16 & $\begin{array}{l}0.71 \\
(0.37- \\
1.36)\end{array}$ & 0.31 & NA & & $\begin{array}{l}1.96 \\
(0.76- \\
5.02)\end{array}$ & 0.16 & NA & \\
\hline $\begin{array}{l}\text { Period from } \\
\text { lung cancer } \\
\text { diagnosis to } \\
\text { brain meta }\end{array}$ & $\begin{array}{l}0.82 \\
(0.48- \\
1.38)\end{array}$ & 0.46 & $\begin{array}{l}1.17 \\
(0.74- \\
1.84)\end{array}$ & 0.49 & $\begin{array}{l}0.82 \\
(0.59- \\
1.15)\end{array}$ & 0.25 & NA & & NA & & NA & \\
\hline \multicolumn{13}{|l|}{$<12$ months } \\
\hline \multicolumn{13}{|l|}{$\begin{array}{l}\text { Over } 12 \\
\text { months }\end{array}$} \\
\hline $\begin{array}{l}\text { Postoperative } \\
\text { neurological } \\
\text { deficits }\end{array}$ & $\begin{array}{l}3.8 \\
(0.50- \\
28.92)\end{array}$ & 0.19 & $\begin{array}{l}1.79 \\
(0.43- \\
7.37)\end{array}$ & 0.41 & $\begin{array}{l}2.52 \\
(1.32- \\
4.8)\end{array}$ & 0.01 & $\begin{array}{l}4.89 \\
(0.62- \\
38.18)\end{array}$ & 0.13 & NA & & $\begin{array}{l}2.46(1.28- \\
7.72)\end{array}$ & 0.01 \\
\hline \multicolumn{13}{|l|}{ NSCLC } \\
\hline \multirow{5}{*}{$\begin{array}{l}\text { EGFR } \\
\text { mutant } \\
\text { ALK mutant } \\
\text { PD-L1 } \\
\text { positive }\end{array}$} & $\begin{array}{l}0.63 \\
0.34-\end{array}$ & 0.13 & 0.81 & 0.43 & 0.52 & 0.51 & 0.54 & 0.42 & $\begin{array}{l}1.67 \\
0.53-\end{array}$ & 0.37 & \multirow{5}{*}{$\begin{array}{l}0.73(0.30- \\
1.77) \\
0.80(0.25- \\
2.52) \\
\mathbf{2 . 2 6}(0.9- \\
\mathbf{5 . 6 2})\end{array}$} & 0.49 \\
\hline & $1.15)$ & 0.95 & $1.37)$ & 0.88 & $3.76)$ & 0.96 & $2.44)$ & & $5.18)$ & 0.98 & & 0.70 \\
\hline & 0.95 & 0.7 & 1.06 & 0.94 & 0.98 & 0.08 & 7.2 & 0.49 & 1.01 & 0.94 & & 0.08 \\
\hline & $\begin{array}{l}(0.32- \\
2.79)\end{array}$ & & $2.37)$ & & $\begin{array}{l}(0.52- \\
1.86)\end{array}$ & & $52.75)$ & & $\begin{array}{l}(0.22- \\
4.57)\end{array}$ & & & \\
\hline & $\begin{array}{l}0.80 \\
(0.26- \\
2.4)\end{array}$ & & $\begin{array}{l}1.03 \\
(0.39- \\
2.71)\end{array}$ & & $\begin{array}{l}2.1 \\
(0.9- \\
4.89)\end{array}$ & & $\begin{array}{l}0.6 \\
(0.15- \\
2.51)\end{array}$ & & $\begin{array}{l}1.03 \\
(0.94- \\
1.03)\end{array}$ & & & \\
\hline
\end{tabular}

NSCLC: non-small cell lung cancer; SCLC: small cell lung cancer; PFS, progression-free survival; HR: hazard ratio; Cl: confidence interval; WBRT: whole brain radiation therapy; EGFR: epidermal growth factor receptor; ALK: anaplastic lymphoma kinase; PD-L1: programmed death ligand-1 


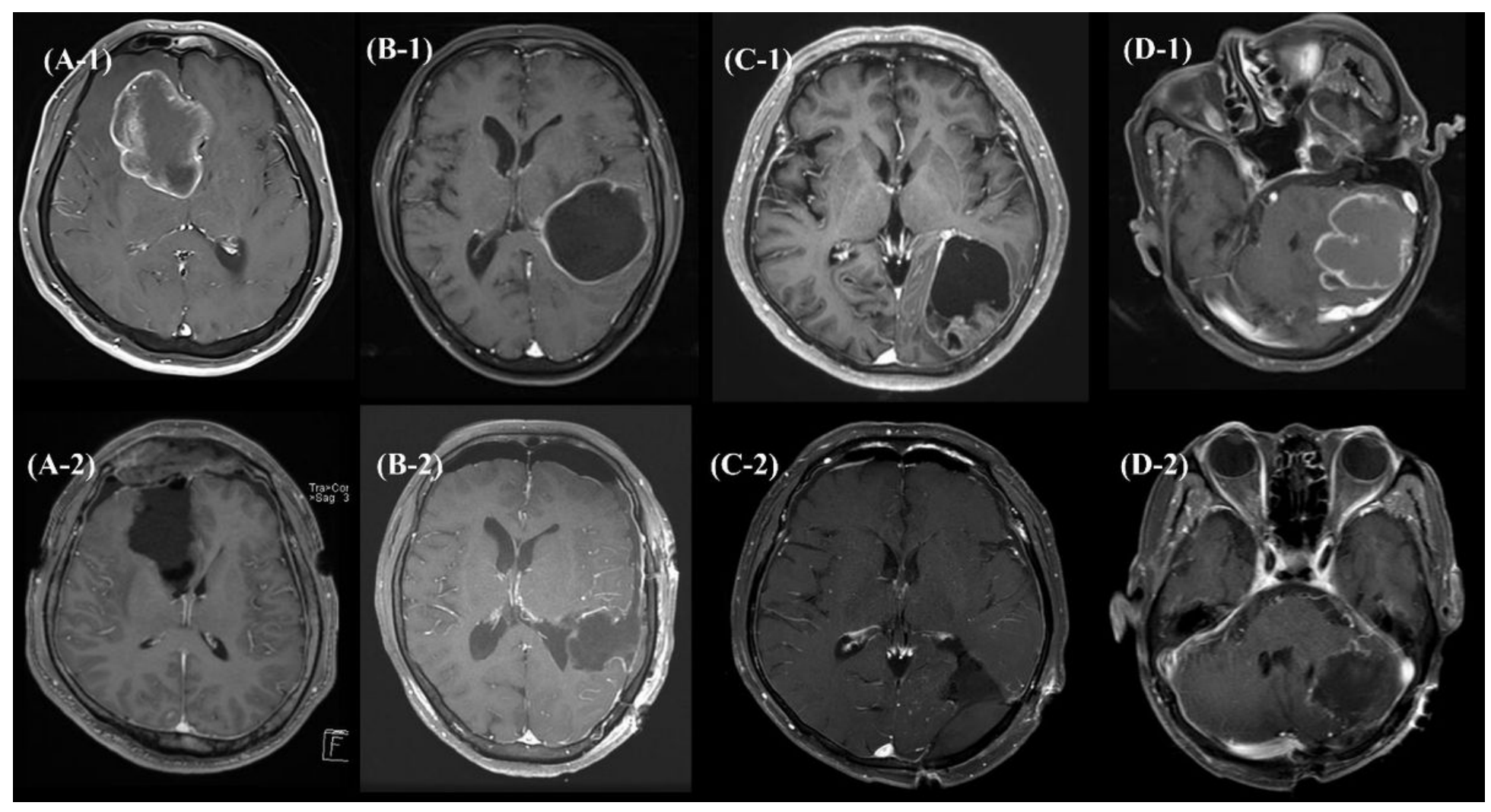

\section{Figure 1}

Representative radiological findings for brain metastasis from lung cancer (A-1,2) A 52-year-old male patient with non-small cell lung cancer (NSCLC)-squamous cell carcinoma. A large right frontal lobe tumor is resected to relieve intracranial hypertension. Other lesions were treated by gamma knife radiosurgery. (B-1,2) A 65-year-old male patient with NSCLC-adenocarcinoma. Left temporal lobe large cystic tumor is resected totally. Another small right parietal tumor is treated by gamma knife radiosurgery. $(\mathrm{C}-1,2) \mathrm{A} 70$-year-old male patient with NSCLC adenocarcinoma. Single left occipital solid and the cystic tumor is resected. Postoperative homonymous hemianopsia is developed. The patient has not received adjuvant therapy. (D-1,2) A 79-year-old male patient with NSCLC squamous cell carcinoma. A large cystic cerebellar tumor is resected to relieve obstructive hydrocephalus. Postoperatively, the fourth ventricle is decompressed and hydrocephalus is relieved. 


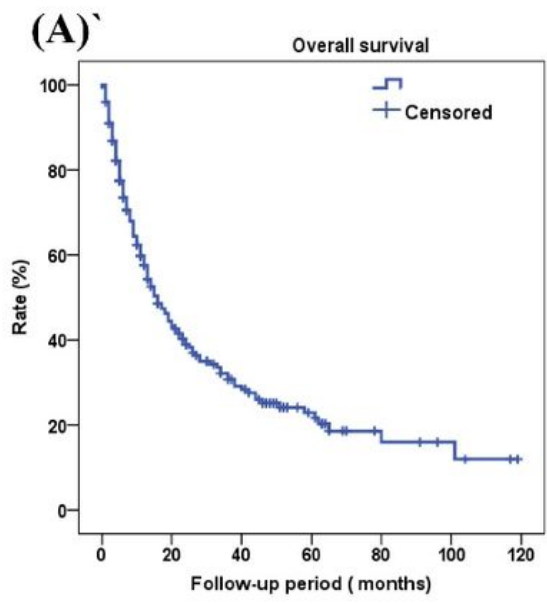

\section{Overall survival}

1-year OS: 57\%, 2-year OS: $40 \%$ 3-year OS: 30\%, 4-year OS: $25 \%$

(D)

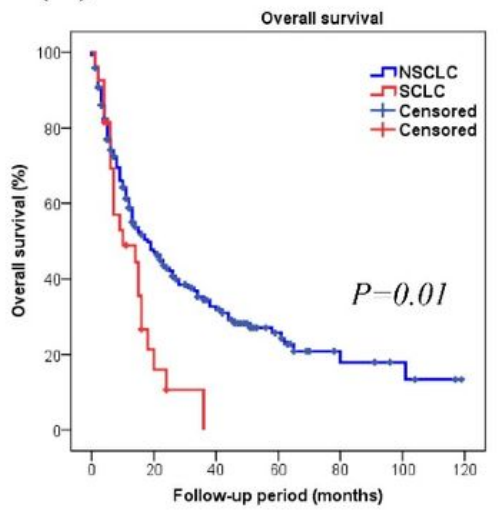

\section{NSCLC group, OS}

1-year: $58 \%$, 2-year: $42 \%$, 3-year: $34 \%$ SCLC group

1-year: 44\%, 2-year: 10\%, 3-year: 0\%
(B)

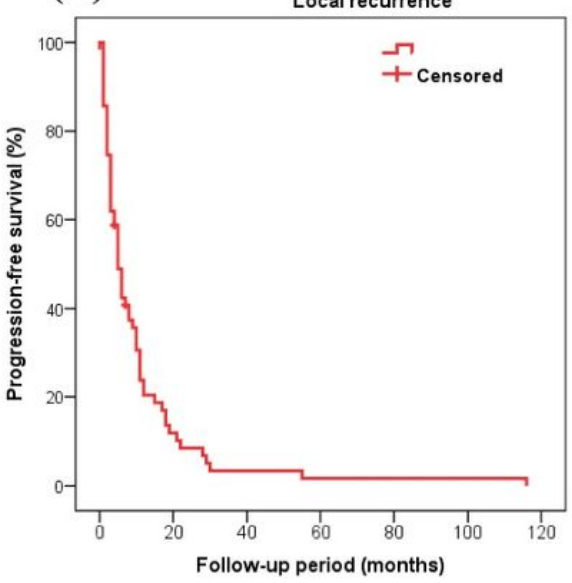

PFS for local recurrence

50\%-PFS: 5 months

6-months: $42 \%$, 1-year: $20 \%$

(E)

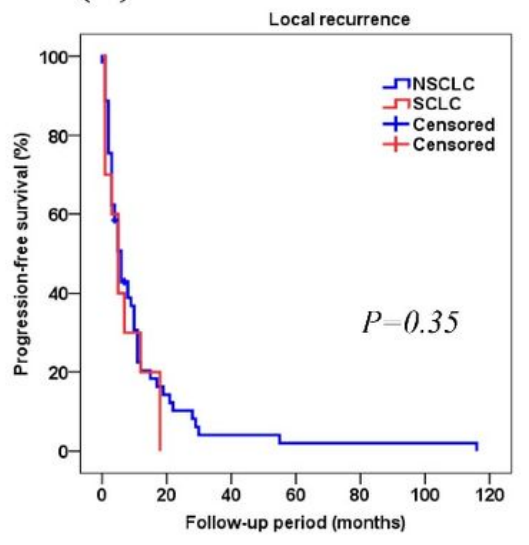

NSCLC group, PFS of local recurrence 6-month: $51 \%$, 1-year: $20 \%$ SCLC group 6-month:36\%, 1-year: 18\%
(C)

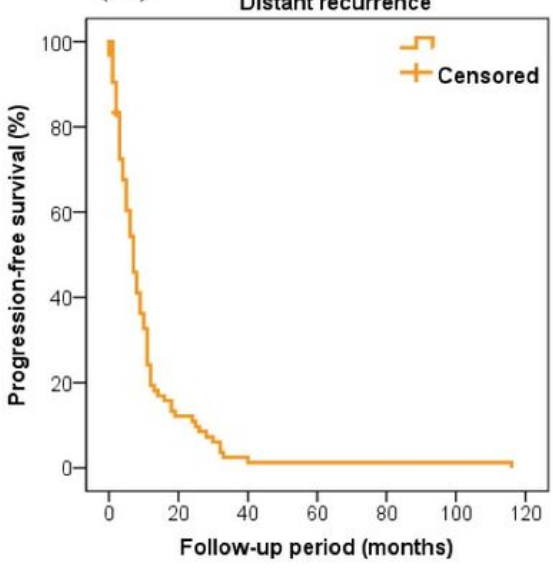

PFS for distant recurrence 50\%-PFS: 7 months 6-month: $54 \%$, 1-year: $24 \%$

(F)

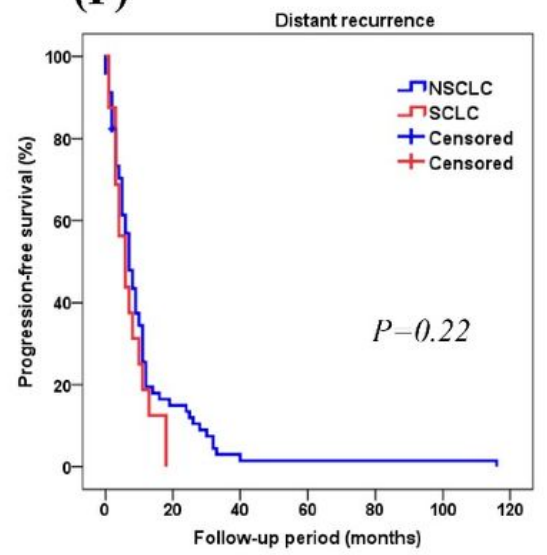

NSCLC group, PFS for distant recurrence 6-month: $56 \%$, 1-year: $19 \%$

SCLC group

6-month: $56 \%$, 1-year: $18 \%$

\section{Figure 2}

Kaplan-Meier survival analysis of the enrolled patients (A) Overall survival (OS) of all surgically treated patients with lung cancer brain metastasis. (B) Progression-free survival (PFS) of local recurrence of all surgically treated patients with lung cancer brain metastasis. (C) PFS of distant recurrence of all of the surgically treated patients with lung cancer brain metastasis. (D) The difference of OS between non-small cell lung cancer (NSCLC) and small cell lung cancer (SCLC) groups. NSCLC group shows better overall survival after surgical resection of brain metastasis. (E) The difference of PFS for local recurrence between NSCLC and SCLC group. There is no significant PFS difference. (F) The difference of PFS for distant recurrence between NSCLC and SCLC group. There is no significant PFS difference. 
(A)

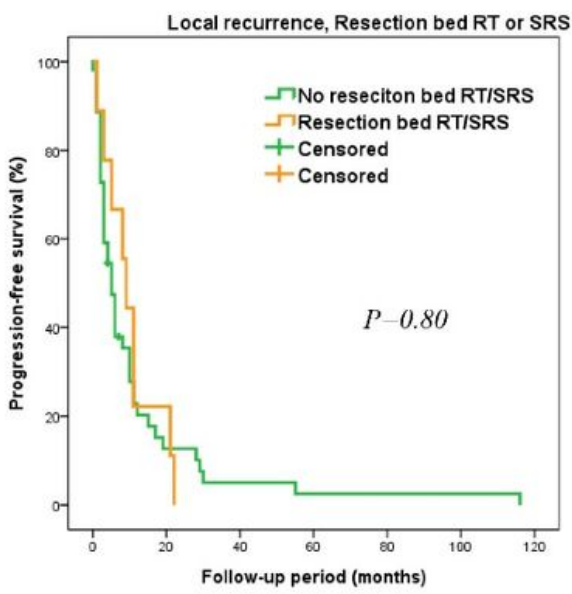

\section{NSCLC group}

No RT/SRS group, 6-month PFS:38\%, 1-year PFS: 20\%

RT/SRS group, 6-month PFS: 66\%, 1-year PFS: 22\%

(C)

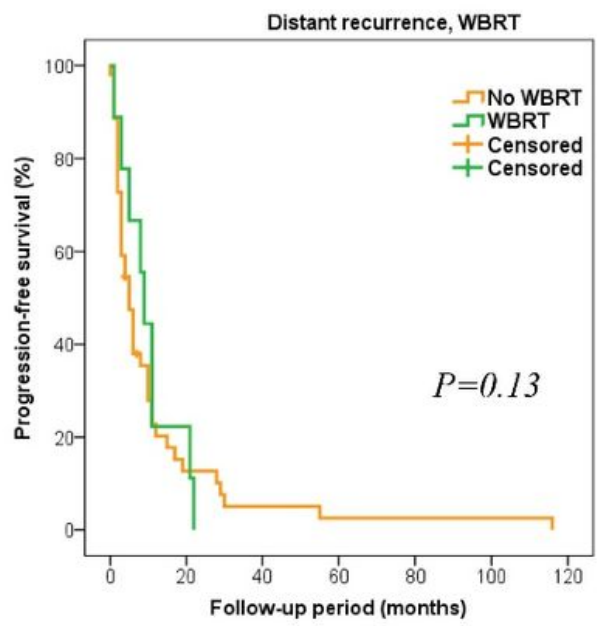

NSCLC and SCLC group, Whole-brain irradiation No RT group, 6-month PFS:54\%, 1-year PFS: 20\% RT group, 6-month PFS: $60 \%$
(B)

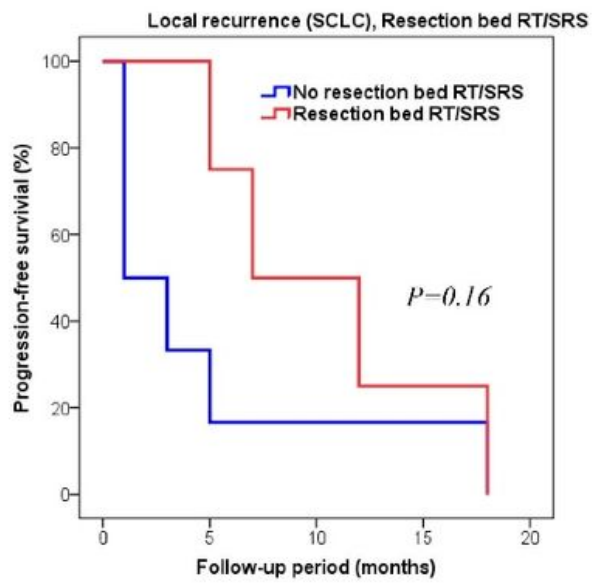

SCLC group

No RT/SRS group, 6-month PFS: 14\%, 1-year PFS: 14\%

RT/SRS group, 6-month PFS: 75\%, 1-year PFS: 25\%

(D)

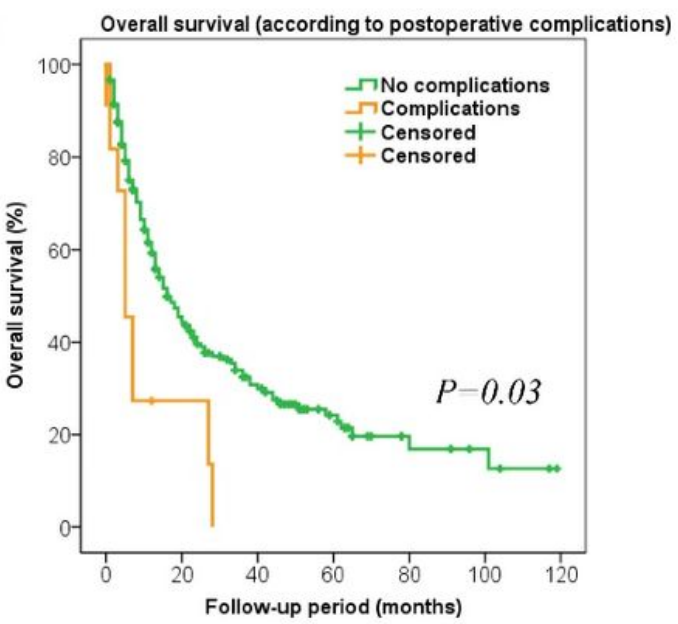

NSCLC and SCLC group

No complication group, 6-month 99\%, 1-year: 59\%, 2-year: 39\%

Complication group 6-month: $45 \%$, 1-year: $27 \%$

\section{Figure 3}

Kaplan-Meier survival analysis of the enrolled patients (A) The difference of progression-free survival (PFS) for local recurrence for resection bed adjuvant radiotherapy in the NSCLC group. There is no statistically significant PFS difference. (B) The difference of PFS for local recurrence for resection bed adjuvant radiotherapy in SCLC group. The adjuvant radiotherapy group shows better PFS for local recurrence than no adjuvant radiotherapy group. However, this result was statistically insignificant. (C) The difference of PFS for distant recurrence regarding adjuvant whole-brain irradiation (WBRT). There is no statistically significant difference in PFS for distant recurrence. (D) The development of postoperative complications affects the overall survival of lung cancer brain metastasis. 

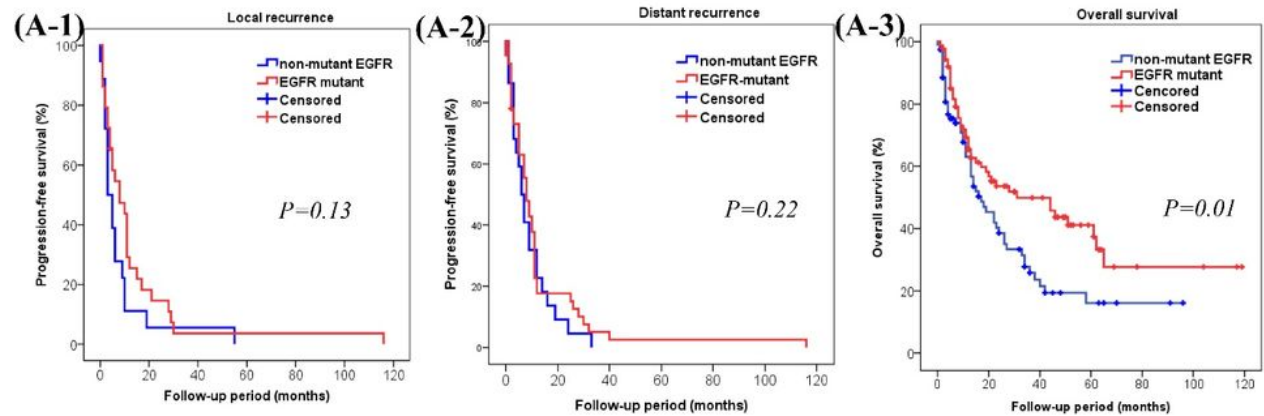

In NSCLC group, PFS for local recurrence EGFR (-), 6-month: 29\%, 1-year: 11\% In NSCLC group, PFS for distant recur EGFR (-), 6-month: 56\%, 1 yr PFS: $25 \%$ EGFR (+): 6 mo PFS: $56 \%, 1$ yr PFS: $18 \%$

In NSCLC group, Overall survival EGFR (-): lyr OS: $62 \%, 2$ yr OS: $38 \%$ EGFR (+), 6-month: 54\%, 1-year: 25\%
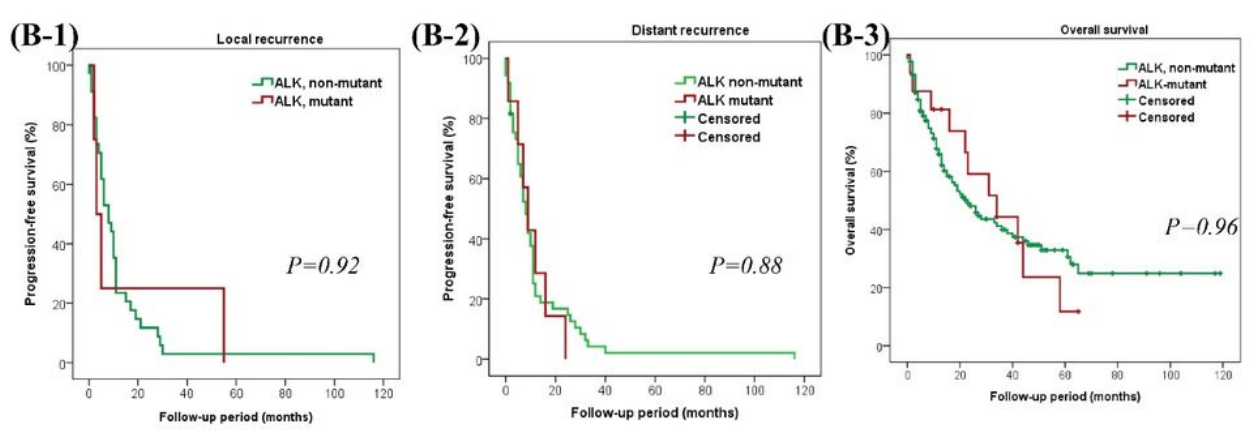

In NSCLC group, PFS for local recurrence ALK (-), 6-month: $82 \%, 12$-month: $23 \%$ ALK (+), 6-month: $25 \%$, 12-month: $25 \%$

(C-1)

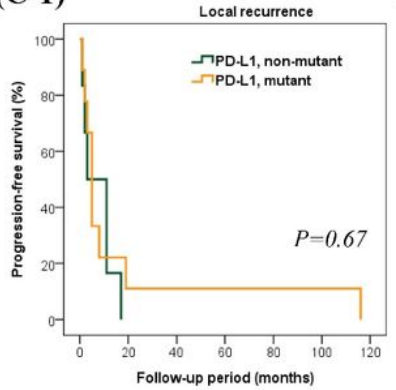

In NSCLC group, PFS for local recurre PD L1 (-), 6-month:50\%, 1-year: 16\% PD L1 (+), 6-month: 33\%, 1-year: $22 \%$
In NSCLC group, PFS for distant recurrence ALK (-), 6-month: $82 \%$, 1-year: $23 \%$ (+), 6-month: 71\%, 1-year: $28 \%$

(C-2)

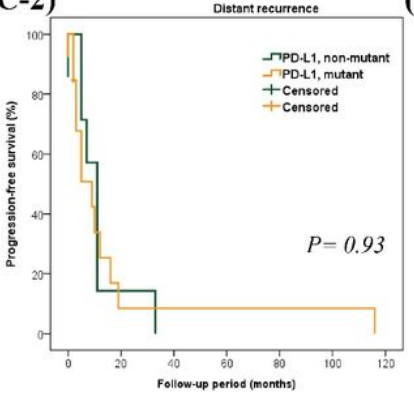

In NSCLC group, PFS for distant recur
PD L1 (-), 6-month:57\%, 1-year: 14\% PD L1 (+), 6-month: 50\%, 1-year: $25 \%$
In NSCLC group, Overall survival

ALK (-), 6-month: 79\%, 1-year: 65\% 2-year: $48 \%$ (+), 6-month: $87 \%$, 1-year: $81 \%$ 2-year: $59 \%$

(C-3)

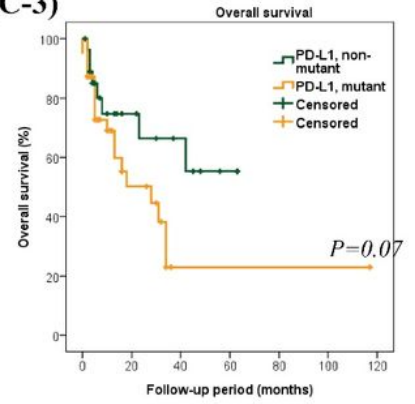

In NSCLC group, Overall survival PD L. (-), 1-year:74\%, 2-year: $66 \%$ PD L1 (+), 1-year: 69\%, 2-year: $50 \%$

\section{Figure 4}

Kaplan-Meier survival analysis of the enrolled patients Overall survival (OS), progression-free survival (PFS) for local and distant recurrence according to immunohistochemical findings of non-small cell lung carcinoma (NSCLC). $(A-1,2,3)$ The OS and PFS difference according to epidermal growth factor receptor (EGFR) mutation in the NSCLC group. There was no significant difference in PFS for local and distant recurrence. However, the EGFR mutant group showed better OS than EGFR non-mutant group. (B-1,2,3) The OS and PFS difference according to anaplastic lymphoma kinase-1 (ALK-1) mutation in the NSCLC group. There was no significant difference in OS, PFS for local and distant recurrence according to ALK-1 mutation. (C-1,2,3) The OS and PFS difference according to programmed deathligand 1 (PD-L1) mutation in the NSCLC group. There is no significant difference in OS, PFS for local and distant recurrence according to ALK-1 mutation. (PD-L1 expression rate above 20\%). 